$17^{\text {th }}$ International Congress of Metrology, 09006 (2015)

DOI: $10.1051 /$ metrology / 201509006

(C) Owned by the authors, published by EDP Sciences, 2015

\title{
Incertitudes liées au calcul de dose annuelle, le rôle des données d'entrées
}

A. Albrecht*, R. Champion**, S. Dinant**, L.Gouriou*, G. Pépin*, V.Schneider**

ANDRA, * 1-7 rue Jean-Monnet 92298 Châtenay-Malabry cedex

ANDRA, ** CSA, BP 7, 10200 Soulaines d'huys

\begin{abstract}
Within the regulatory framework, basic nuclear installations have to estimate the annual radiological impact. This is of particular importance for the aquatic pathway, where release fluxes are more important than for the atmospheric pathway. An estimation of uncertainties associated with these calculations have recently become a requirement.
\end{abstract}

In the case of the Andra Centre Aube disposal facility for low and intermediate, short-lived radioactive waste, the elaboration process for dose estimations is carried out in 3 assessment stages, namely the estimation of:

radionuclide release (input data),

riverine concentrations,

biosphere transfer and related doses to reference groups.

In all cases output uncertainties are used in the next modelling step together with probability distributions for model-specific parameter values.

After a presentation of the contents of the 3 stages, this contribution discusses in detail the estimation of radionuclide releases (input parameters) based on on-site measurements, such as continuous monitoring or laboratory radiochemical analyses.

The characterization of the flow measurements and activities of radionuclides are presented by taking into account every instrumental and analytical contribution and especially the contribution due to the variability of the sample. An additional example for the improvement of uncertainties related to river flow is developed.

Finally, the contribution of the input parameters on the final evaluation of radiological impact for the aquatic pathway is described by presenting limiting factors and elements to be improved.

\section{Contexte et objectif}

Le Centre de Stockage de l'Aube (CSA) de l'Andra, est une Installation Nucléaire de Base (INB) qui a pour vocation de stocker des déchets nucléaires de faible et moyenne activité à vie courte. Le CSA est tenu de dresser un bilan annuel de surveillance de l'environnement à partir des données d'entrée de surveillance.[1]

Dans ce cadre, l'Autorité de Sûreté Nucléaire demande aux INB d'évaluer les principaux facteurs d'incertitudes associées au calcul de dose [2].

Au CSA, cette évaluation est complétée par une quantification des facteurs concourant le plus à l'incertitude de la dose, sur la base d'une analyse de sensibilité multiparamétrique.

Les indicateurs retenus pour l'évaluation sont la concentration à la rivière des Noues d'Amance et la dose pour les populations du groupe agricole. Le choix de considérer un indicateur intermédiaire (concentration à la rivière) afin de ne pas "mélanger» dans l'analyse les paramètres de transfert par l'eau avec ceux du compartiment biosphère a nécessité un découpage de l'étude en 3 étapes:

- Une caractérisation des incertitudes des données d'entrée en particulier le volume rejeté du bassin d'orage, le débit des Noues d'Amance et l'activité des radionucléides;

- Une étude multiparamétrique relative au transfert par l'eau, du bassin d'orage à la rivière des Noues d'Amance, avec pour indicateur intermédiaire la concentration des radionucléides dans la rivière ;

- Une étude multiparamétrique relative au modèle biosphère d'évaluation de la dose, avec pour indicateur final la dose pour les populations adultes du groupe agricole.

Dans ce qui suit, l'étape 1 de caractérisation des incertitudes des données d'entrées est principalement détaillée.

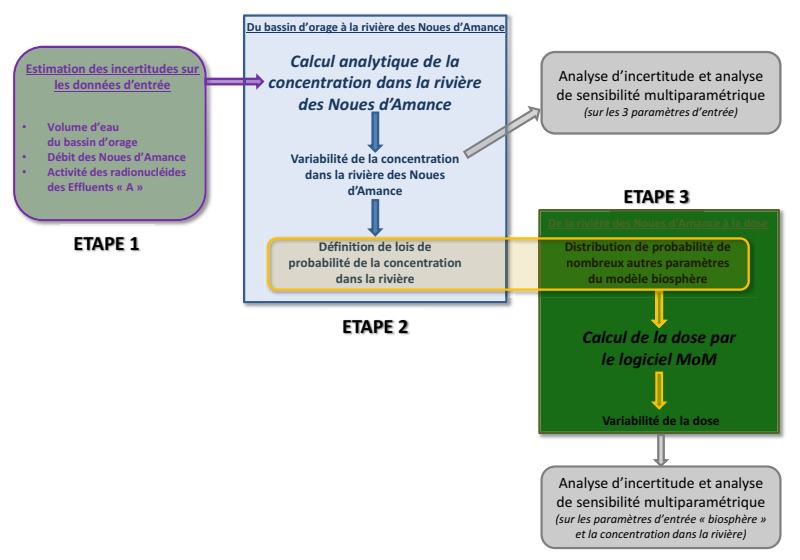

Figure 1 - Les différentes étapes de l'étude

This is an Open Access article distributed under the terms of the Creative Commons Attribution License 4.0, which permits unrestricted use, distribution, and reproduction in any medium, provided the original work is properly cited. 
Ce document présente de manière factuelle l'ensemble des travaux des analyses multiparamétriques menées, en incluant les modalités et les hypothèses de l'étude, puis les résultats.

\section{Il est structuré comme suit :}

Le chapitre 2 présente la caractérisation des incertitudes menée sur les données d'entrée ; en tenant compte dans chaque cas de l'incertitude instrumentale, analytique et surtout de la variabilité de l'échantillon ;

Le chapitre 3 traite des modalités de l'évaluation, en posant le cadre général des évaluations (les indicateurs intermédiaires et finaux retenus, les radionucléides retenus, l'évaluation analytique de la concentration et de la dose), et enfin la méthodologie et le traitement des sensibilités appliquées à la partie transfert par l'eau (du bassin d'orage à la rivière) et à la partie biosphère.

Le chapitre 4 présente l'analyse multiparamétrique de la partie transfert par l'eau (du bassin d'orage à la rivière) et de la partie biosphère.

Le chapitre 5 est une synthèse générale dégageant les grands enseignements de l'analyse multiparamétrique des calculs de dose dans le cadre des transferts par l'eau à ce stade.

\section{La caractérisation des incertitudes sur les données d'entrée}

Le calcul de la dose pour la voie de transfert par l'eau impose de connaître les caractéristiques d'entrées que sont les volumes et débits d'eau mis en jeu ainsi que la quantité (ou concentration) de radionucléides.

Aussi les paramètres utilisés dans les données d'entrées pour la caractérisation des incertitudes sont :

- Le volume d'eau rejeté issu du bassin d'orage ;

- Le débit du cours d'eau les Noues d'Amance ;

- L'activité des radionucléides dans les effluents liquides industriels.
Les incertitudes sont calculées en s'appuyant sur le Guide pour l'expression des incertitudes de mesures [3].

\section{$2.1 \quad$ Estimation des incertitudes sur le volume d'eau rejeté issu du bassin d'orage du CSA}

2.1.1 Modalités de la gestion des effluents liquides du CSA [4].

Le bassin d'orage du CSA recueille :

- Les eaux usées du site après épuration en station ;

- Les eaux pluviales ;

- Les eaux de la nappe recueillies par infiltration ;

- Les effluents industriels vérifiés et conformes ;

\subsubsection{Instrumentation pour la gestion du rejet au bassin d'orage du CSA}

Le rejet des effluents issus du BO vers les Noues d'Amance est assuré par 4 pompes, 3 pompes de débit nominal de $66 \mathrm{~L} / \mathrm{s}$ (ou pompe grand débit) et 1 pompe de débit nominal de $4 \mathrm{~L} / \mathrm{s}$ (ou pompe petit débit).

Les pompes sont asservies, par une mesure de niveau, au volume total du bassin d'orage. Le volume du bassin d'orage ne peut être inférieur à $10000 \mathrm{~m}^{3}$ et ne peut dépasser $30000 \mathrm{~m}^{3}$. Le fonctionnement des pompes est étagé ; soit la pompe petit débit fonctionne seule, soit une pompe grand débit fonctionne seule, soit deux ou trois pompes grand débit fonctionnent.

En amont de chacune des pompes, un débitmètre électromagnétique [5] est installé. Le volume d'eau rejeté est ainsi mesuré en direct (par lecture du compteur volumique sur les débitmètres) et la valeur est reportée en supervision. La supervision enregistre également le temps de fonctionnement de chaque pompe.

2.1.3 Estimation des incertitudes du volume d'eau rejeté du bassin d'orage du CSA 
Afin de s'affranchir des variations dues à la pluviométrie et d'avoir une vision générale du fonctionnement des pompes, le calcul du volume rejeté est dans un premier temps effectué pour une année complète. Cette valeur est moyennée sur 12 mois. Les facteurs influents repérés sur la valeur du volume d'effluent rejeté proviennent essentiellement :

- de la mesure du volume par les débitmètres ; - du report du signal mesuré en supervision.

Les facteurs estimés comme négligeables sont :

- le temps de déclenchement des pompes (suite à la mesure du niveau) devant la durée de fonctionnement des pompes.

- l'incertitude sur la durée de fonctionnement car la connaissance du signal de coupure ou d'alimentation d'une pompe est bien déterminée par la supervision.

- le temps de transfert jusqu'à la supervision (estimé à environ $10 \mathrm{~s}$ ) très petit comparé à la durée de fonctionnement annuel des pompes.

2.1.3.1 Estimation des incertitudes sur le volume fourni par les débitmètres

Le volume rejeté pour toute une année est calculé à partir de l'occurrence de fonctionnement des pompes. En s'appuyant sur les données du constructeur et la durée de fonctionnement des pompes, le régime de fonctionnement idéal est arbitrairement défini après $5 \mathrm{~min}$ de fonctionnement.

Les volumes rejetés en fonctionnement dégradé $(<5 \mathrm{~min})$ et en fonctionnement idéal (> $5 \mathrm{~min}$ ) sont calculés; pour chacun de ces régimes de fonctionnement, on associe une incertitude respectivement de $0,5 \%$ et $0,1 \%$. Ces incertitudes tiennent compte des données $\mathrm{du}$ constructeur, des variations de température de l'eau sur la mesure du débit et de la dérive des appareils.

2.1.3.2 Estimation des incertitudes dues à la transmission jusqu'à la supervision

A cette incertitude instrumentale, on ajoute une incertitude sur la totalisation du volume rejeté. En effet, on enregistre un écart quotidien entre les valeurs lues en direct sur les débitmètres et les valeurs enregistrées en supervision.
L'écart moyen maximal mensuel est conservé comme incertitude-type sur ce paramètre.

Au final, l'incertitude totale sur le volume du bassin d'orage s'exprime selon :

$$
u_{\text {total volume }}=\sqrt{u_{\text {instrumentale }}^{2}+u_{\text {enregistrement }}^{2}}
$$

L'incertitude sur le volume rejeté est calculée comme présenté ci-dessous :

\begin{tabular}{|c|c|c|c|}
\hline Grandeur & $\begin{array}{c}\text { Origine des } \\
\text { incertitudes }\end{array}$ & Expression des incertitudes & $\begin{array}{c}\text { Incertitu } \\
\text {-de } \\
\text { élargie } \\
(\mathrm{k}=2)\end{array}$ \\
\hline $\begin{array}{c}\text { Volume } \\
\text { rejeté } \mathrm{V} \\
\text { en } \mathrm{m} 3\end{array}$ & $\begin{array}{c}\text { - EMT } \\
\text { vérification } \\
\text { dépétabilité } \\
\text { - écart du } \\
\text { report à la } \\
\text { supervision }\end{array}$ & $\sqrt{(E M T \text { vérif } / 3)^{2}+u_{\text {répétabilité }}^{2}+u_{\text {réport }}^{2}}$ & \\
& $6,2 \%$ & \\
\hline
\end{tabular}

Tableau 1- Estimation des incertitudes sur volume rejeté par le bassin d'orage

L'incertitude finale élargie $(k=2)$ sur le volume rejeté du bassin d'orage du CSA est de 6,2\%.

\subsection{Estimation des incertitudes sur le débit des Noues d'Amance}

\subsubsection{Conditions de la mesure du débit des Noues d'Amance}

Le débit des Noues d'Amance, est mesuré au point référencé $R 2-C D 24$ situé en aval du point de rejet. La station hydrométrique à ce point est équipée [6]:

- d'une échelle limnimétrique de référence,

- d'un puits de mesure qui est relié par une prise d'eau dans la rivière et équipé d'un limnigraphe à flotteur et d'un codeur numérique,

- d'un chenal sous un pont ou sont réalisées périodiquement des mesures du débit du cours d'eau par jaugeage au moulinet de rivière. Ce chenal est considéré comme stable hydrauliquement car le lit de la rivière est bétonné (radier) et les berges sont empierrées.

- d'un venturi à canal ouvert, installé en 2013 pour la mesure des faibles débits, muni d'un puits de mesure

L'échelle limnimétrique, graduée de 0 à $200 \mathrm{~cm}$, est placée en rive droite, juste en amont du pont. 
Le puits de mesure est également situé en amont du pont en rive gauche. II est équipé de deux systèmes d'acquisition des hauteurs d'eau: un limnigraphe qui permet l'enregistrement de la chronique de hauteurs d'eau sur un rouleau en papier et un codeur numérique de type Thalimèdes réglé à un pas temps d'acquisition de $15 \mathrm{~min}$. Ces deux systèmes d'acquisition sont reliés à un dispositif unique de mesure des hauteurs d'eau constitué d'une roue mise en mouvement par un câble équipé d'un flotteur et d'un contre-poids.

Le déstockage des données numériques et le changement de la feuille du limnigraphe sont effectués tous les mois.

Les chroniques brutes de hauteur d'eau enregistrées sont validées :

- à partir des lectures de la hauteur d'eau à l'échelle limnimétrique réalisées: lors des déstockages mensuels du Thalimèdes, lors des jaugeages et lors de visites de contrôle non programmées,

- par comparaison avec la courbe tracée sur le rouleau en papier du limnigraphe,

- en vérifiant si nécessaire la cohérence entre l'allure de la chronique enregistrée avec des événements hydrologiques particuliers : crues, pluviométrie..

Les étapes de suivis du débit du cours d'eau comprennent :

- l'établissement des chroniques de hauteurs d'eau validées à partir des enregistrements,

- la réalisation de jaugeages au point R2-CD24 afin de couvrir annuellement la gamme des hauteurs d'eau de la rivière,

- le contrôle et la mise à jour de la courbe d'étalonnage de la rivière (relation hauteur/débit),

- et le calcul des chroniques de débits par la relation «hauteur/débit » établie précédemment.
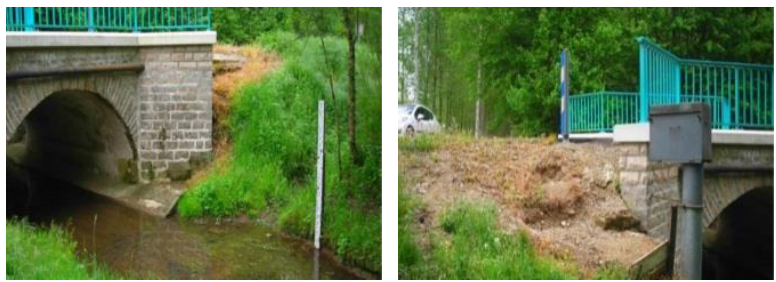

Figure 2 - Position des capteurs et enregistreurs de débit au point R2-CD24 [6]

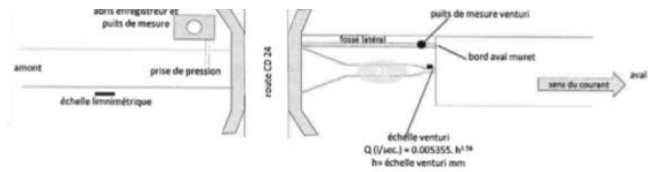

Le débit du cours d'eau au point R2- CD24 est suivi depuis 1985. Cependant, entre 1985 et 1990 le suivi et le nombre de jaugeage est limité à 1 minimum.

Afin d'avoir les variations instrumentales et une estimation bien représentative du débit du cours d'eau et des variations instrumentales, toutes les données de débit enregistrées entre 2002 et 2012 sont exploitées.

\subsubsection{Estimation des incertitudes du débit des Noues d'Amance}

Les facteurs influents sur la mesure du débit sont les instruments de mesure (leur étalonnage, leur dérive et leur fidélité), et surtout les fluctuations géométriques du profil du cours d'eau.

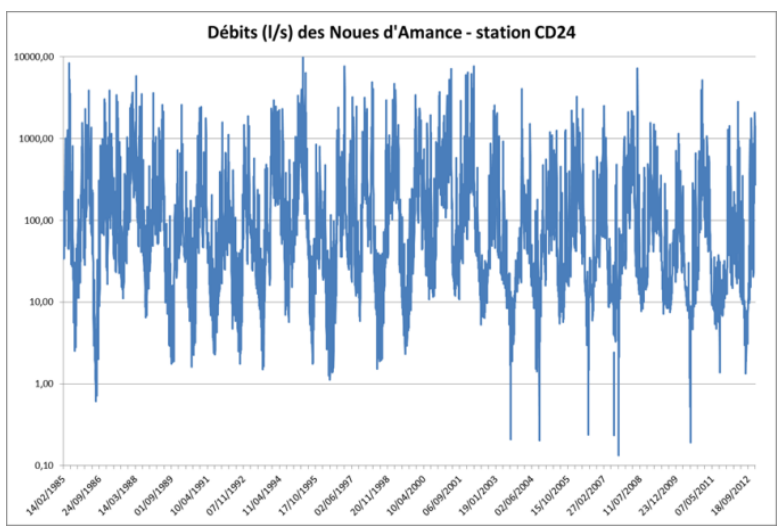

Figure 3 - Chronique journalière des mesures de débit des Noues d'Amance entre 1985 et 2012 en $L / s$ au point CD24

L'étude des chroniques de débit des Noues d'Amance entre 1985 et 2012 (Cf. Figure 3), montre des variations de débit périodiques et une moyenne «intuitive » du débit autour de $100 \mathrm{~L} / \mathrm{s}$.

Sur la Figure 4, on observe une moyenne intuitive de débit annuel comprise entre 400 et $600 \mathrm{~L} / \mathrm{s}$ entre 1985 et 2001 , cette moyenne diminue depuis 2002 , elle est comprise autour de $200 \mathrm{~L} / \mathrm{s}$. 


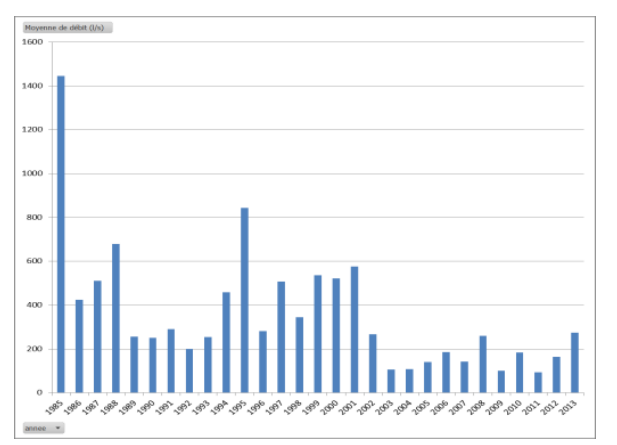

Figure 4- Moyenne annuelle des mesures de débit des Noues d'Amance de 1985 à 2012

L'étude des différentes chroniques ont permis de montrer l'amélioration des mesures apportée par l'installation du venturi. En effet, $40 \%$ du temps les faibles débits sont mesurés par le venturi (débits inférieurs à $0,5 \mathrm{l} / \mathrm{s}$ ).

Afin de tenir compte, de façon globale, des facteurs influents, la moyenne des débits entre 2002 et 2012 est retenue, celle-ci étant calculée à partir de 96 mesures quotidiennes. Avant 2002, la fréquence des mesures n'est en effet pas homogène. L'étendue des variations quotidiennes est représentée par l'écart-type des 96 mesures quotidiennes. Pour représenter les variations annuelles, la moyenne des écarts-types quotidiens est calculée sur cette période.

On estime que cette moyenne est représentative de tous les facteurs influents (instruments et milieu) et de leur variation au cours du temps.

L'incertitude élargie du débit des Noues d'Amance est estimée à $18 \%(k=2)$ incluant une incertitude instrumentale de $5 \%$.

\subsection{Estimation des incertitudes sur l'activité des radionucléides dans les effluents industriels}

\subsubsection{Rappel des modalités de la gestion des effluents industriels du CSA}

Ces effluents sont collectés dans des cuves en fonction de leur provenance (bâtiment des services, atelier de conditionnement des déchets, bâtiment mécanique ou bâtiment de transit).
Un suivi radiologique de ces effluents est effectué avant chaque rejet afin de vérifier le respect de l'autorisation des conditions de rejet avant déversement au bassin d'orage.

Les contrôles radiologiques portent sur la détermination des activités volumiques (en $\mathrm{Bq} / \mathrm{L}$ ) en émetteurs alpha et en émetteurs bêta et gamma, $\mathrm{H}-3$ et $\mathrm{C}-14$.

Le laboratoire d'analyses radiologiques, qui est agréé pour ces analyses, utilise les techniques suivantes :

- Spectrométrie $\alpha$;

- Spectrométrie $\mathrm{\gamma}$;

- Scintillation liquide (H-3, C-14).

\subsubsection{Estimation des incertitudes sur les activités des radionucléides des effluents industriels}

L'estimation des incertitudes est découpée en 2 parties, la partie analytique et la partie échantillonnage.

Pour la partie analytique, les incertitudes correspondent à celles rendues par le laboratoire pour chaque type d'analyse radiologique. Le laboratoire estime et développe ses incertitudes d'analyse selon la norme ISO 11929 [7]. L'incertitude d'analyse correspond à celle mesurée sur l'échantillon prélevé.

Lorsque le résultat d'analyse est inférieur au seuil de décision SD, l'incertitude est prise égale à $100 \%$.

Pour l'échantillonnage, l'estimation des incertitudes repose sur la détermination de l'incertitude du volume des cuves. Les cuves sont remplies à $95 \%$ et vidées lorsque ce volume est atteint. Jusqu'à présent, par précaution, l'activité totale par catégorie de radionucléide est calculée en tenant compte du volume nominal pour chaque cuve.

On estime donc que le volume de la cuve est compris entre $100 \%$ et $90 \%$, l'incertitude $(u)$ sur ce volume est estimée à partir d'une loi uniforme :

$$
u_{\text {volume cuve }}=\frac{0.1 * \text { volume }_{\text {cuve }}}{\sqrt{3}}
$$


Pour chaque cuve d'effluents industriels, l'activité totale par catégorie de radionucléide est calculée comme suit : activité volumique $(B q / L) *$ volume d'effluents $(L)$.

L'activité totale annuelle est la somme des valeurs, les variances peuvent s'additionner; l'incertitude est alors la racine carrée de la somme des variances pour chaque catégories de radionucléides et pour chaque niveau considéré.

La valeur d'incertitude sur l'activité volumique est l'incertitude type; lorsque l'activité volumique est inférieure au seuil de décision (SD), alors l'incertitude est prise égale à $100 \%$.

\section{Les modalités de l'évaluation}

\subsection{Le cadre général}

\subsubsection{Les radionucléides retenus}

Les radionucléides retenus sont ceux dont l'impact est évalué pour les bilans annuels d'exploitation. II s'agit des radionucléides suivants : le $\mathrm{H}-3$, le C-14, le Pu-239 (considéré comme représentatif des émetteurs alpha) et le I-129 (considéré comme représentatif des émetteurs bêta-gamma).

\subsubsection{Le modèle de calcul de la concentration dans la rivière}

Le calcul analytique de la concentration dans la rivière communément utilisé pour les évaluations de dose dans le cadre des bilans annuels, est basé sur des données mesurées au CSA (volume du bassin d'orage, débit des Noues d'Amance et activités des radionucléides).

On suppose une conservation de la masse entre les deux réservoirs : le bassin d'orage et la rivière des Noues d'Amance, en négligeant notamment la décroissance radioactive ; ainsi :

Equivaut à :

$$
\operatorname{Conc}_{B O} \times \operatorname{Vol}_{B O}=\operatorname{Conc}_{N A} \times\left(\operatorname{Vol}_{N A}\right)
$$

Soit :

$$
\operatorname{Conc}_{R N}=\operatorname{Conc}_{B O} \times \text { Taux }_{\text {dilution }}
$$

Avec:

$$
\begin{array}{r}
\operatorname{Conc}_{B O}=\frac{A c t_{R N}}{\operatorname{Vol}_{B O} \times 1000} \\
\text { Et Taux dilution }=\frac{\operatorname{vol}_{B O}}{\operatorname{Vol}_{N A}}
\end{array}
$$

Où :

- $\mathrm{n}_{\text {во }}$ est la quantité de matière dans le réservoir «bassin d'orage » $(\mathrm{mol})$;

- $n_{N A}$ est la quantité de matière dans le réservoir «rivière des Noues d'Amance » (mol) ;

- Conc $_{\mathrm{NA}}$ est la concentration en radionucléide

« $\mathrm{RN}$ » dans la rivière $(\mathrm{Bq} / \mathrm{L})$;

- Conc $_{\text {во }}$ est la concentration en radionucléide

« RN » dans le bassin d'orage (Bq/L) ;

- Taux $_{\text {dilution }}$ est le taux de dilution entre le bassin d'orage et la rivière des Noues d'Amance (-) ;

- $\mathrm{Act}_{\mathrm{RN}}$ est l'activité du radionucléide « RN » mesurée dans les effluents industriels du bassin d'orage $(\mathrm{Bq})$;

- Vol ${ }_{\text {во }}$ est le volume de rejet du bassin d'orage $\left(\mathrm{m}^{3}\right)$;

- Vol ${ }_{\mathrm{NA}}$ est le volume des Noues d'Amance $\left(\mathrm{m}^{3}\right)$. Il est évalué sur la base du débit des Noues d'Amance $Q_{N A}(L / s)$, rapporté sur 1 an.

\subsubsection{Le modèle de calcul de la dose (compartiment biosphère) [8]}

Les expositions font partie des évaluations de transfert qui dans leur globalité peuvent regrouper les quatre voies de contamination principales (cf. Figure 5): l'eau de puits captant un aquifère profond contaminé, l'eau de rivière contaminée par l'eau de surface ou d'un aquifère de surface, une remontée verticale depuis une source plus ou moins profonde et la contamination en lien avec les rejets atmosphériques.

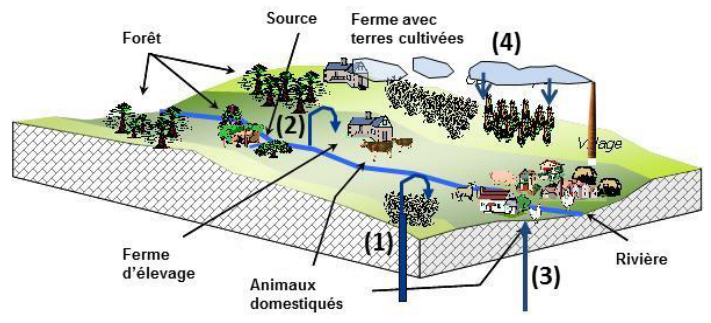

Figure 5 - Illustration de la voie de contamination par la rivière (2) en comparaison avec la voie aquatique "eau de nappe" (1), le remontée (3) et le dépôt 
atmosphérique (4) dans un contexte de paysage agricole

La contamination des plantes représente l'entrée de la chaîne alimentaire qui est considérée dans sa globalité avec les produits agricoles primaires (légumes feuille et racine ; pommes de terres ; fruits ; viandes de porc, de volaille, de vache, d'agneau, lait, œufs) et secondaires tels que les produits laitiers.

L'ensemble des voies de contamination est synthétisé dans la Figure 6 . Les transferts sont quantifiés dans les modèles Aquabios et AquaC_14 [10] soit par des flux (Q, i.e. eau de boisson), des facteurs de transfert (FT : I, Pu) soit par une approche de rapport isotopique (RI : C-14, H-3). Le groupe de référence est un villageois adulte tel que défini pour les analyses de sureté du CSA [9].

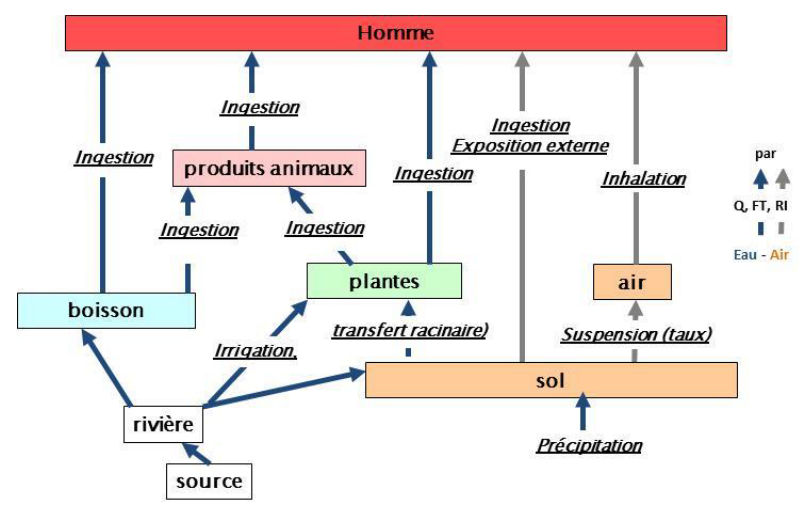

Figure 6 - Synthèse des voies de contamination et relation entre les compartiments contaminés par l'irrigation avec l'eau de rivière et par l'utilisation de celle-ci pour la boisson et l'abreuvement

\subsection{Les méthodologies retenues pour les analyses multiparamétriques}

\subsubsection{La méthodologie retenue pour le calcul de la concentration aux Noues d'Amance}

\subsubsection{Le schéma général}

Le schéma général de mise en œuvre de l'analyse de sensibilité multiparamétrique comprend plusieurs étapes, comme indiqué à la Figure 7, et dont chaque étape est décrite ci-après :

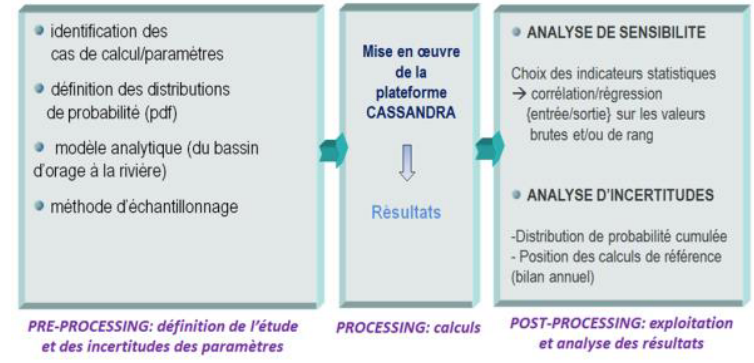

Figure 7 - Schéma général de mise en cuvre de l'étude multiparamétrique

- L'identification des différents paramètres concourant à la concentration dans la rivière des Noues d'Amance à considérer dans les évaluations ;

- La définition des domaines de variabilité possibles des différents paramètres, sous forme de distributions de probabilité continue, bornées par des valeurs minimales et maximales possibles et encadrant de manière symétrique la valeur de référence du bilan annuel d'exploitation ;

- Un échantillonnage des distributions de probabilité pour établir le plan d'expériences couvrant au mieux le spectre de variation multiparamétrique ;

- Dans cette étude, aucune corrélation n'a été imposée: les paramètres d'entrée et les variables de calcul sont considérés comme indépendants ;

- La quantification des indicateurs, en exploitant les outils de simulation de l'Agence ;

- L'analyse d'incertitudes et de sensibilité exploitant différents types d'indicateurs statistiques sur les concentrations dans les Noues d'Amance, et répondant aux différents objectifs.

\subsubsection{La méthode d'échantillonnage retenue}

La méthode d'échantillonnage retenue est la méthode de type HyperCube Latin (méthode dite «LHS», en acronyme anglais). Cette méthode est préférée par l'Andra aux méthodes de tirage aléatoires ou d'importance, puisqu'elle permet de balayer de manière homogène le spectre de variation multiparamétrique des paramètres considérés. Son principe consiste à découper chaque distribution de probabilité en strates équiprobables, et à procéder à un tirage dans au moins chaque (ligne/colonne). 


\subsubsection{La méthodologie retenue pour l'analyse de sensibilité multiparamétrique du calcul de dose}

Le transfert des radionucléides dans la biosphère comprend la migration des radionucléides depuis la rivière des Noues d'Amance jusqu'à l'homme. La quantification de ces transferts dans le modèle de biosphère requiert plusieurs paramètres assortis d'incertitudes. Afin de quantifier l'ensemble de ces incertitudes dans le modèle, il est retenu une démarche similaire à celle traitée dans le $\S$ 3.2.1.

Le retour d'expérience sur ces sujets montre que compte tenu du nombre de paramètres étudiés, 4000 à 5000 échantillons suffisent pour couvrir de manière satisfaisante le spectre de variation multiparamétrique.

Ainsi, pour ce qui concerne le choix des lois de distribution, il est retenu dans le cas d'un manque de données, la loi uniforme ; et dans le cas où le nombre de données est suffisant, une loi triangulaire. Dans le cas spécifique des paramètres composites tel que le coefficient de partage $(\mathrm{Kd})$ ou lorsque les paramètres de transfert qui représentent le produit de deux paramètres suivent des lois normales (par exemple, la concentration en radionucléides sur le solide et en solution pour le $\mathrm{Kd}$ ), une loi Log normale est appliquée.

Afin de déterminer les paramètres les plus influents sur la dose, il est retenu les corrélations de Pearson, calculées sur la base des données brutes, ainsi que les corrélations de Spearman, qui sont basées sur les rangs des valeurs de paramètres.

L'incertitude de la concentration au niveau de la rivière s'est basée pour le calcul d'incertitude global, soit sur une distribution linéaire avec considération de minima et maxima, soit sur une distribution normale construite par l'utilisation des 200 échantillons analysés.

\subsection{Variabilité retenue pour les données d'entrées}

\subsubsection{Données d'entrées pour la voie eau}

Les données d'entrées pour la voie de transfert pour la voie eau sont :

- le volume du BO, la valeur de référence pour cette étude est celle du bilan annuel de 2012
- le débit de Noues d'Amance, la valeur de référence s'appuie sur la moyenne de 2006 à 2012

- l'activité rejetée pour chaque radionucléide, la valeur de référence utilisée est celle du bilan de 2012.

Afin de déterminer la variabilité de chacune des données d'entrées, une loi uniforme est appliquée sur la valeur de référence.

\subsubsection{Données d'entrée pour la biosphère}

La concentration dans les Noues d'Amance est un paramètre dynamique d'entrée des modèles biosphère (Aquabios pour $\mathrm{H}-3, \mathrm{l}-129$ et $\mathrm{Pu}-239$ et AquaC_14 pour le C-14). Afin de pouvoir lui affecter une incertitude, deux méthodes ont été mises en œuvre, la première basée sur une loi uniforme, la seconde sur une loi normale.

La représentation en histogramme des 200 échantillons de concentration en radionucléides montre que les valeurs ne s'ajustent ni à une loi uniforme, ni à une loi normale. L'utilisation des deux lois parait donc une approche prudente pour la considération de l'incertitude de la concentration des radionucléides dans les Noues d'Amance dans l'évaluation d'incertitude globale.

3.3.3 Les incertitudes des paramètres biosphère: comportement du groupe de référence et transfert des radionucléides dans les compartiments

Pour une estimation de l'incertitude par approche Monte-Carlo (stochastique), un grand nombre de paramètres doit être renseigné pour quantifier l'incertitude des paramètres influents. Le choix de paramètres à considérer est basé sur l'expérience acquise lors des 12 dernières années [11] [14]. Les études stochastiques réalisées à l'agence ont permis la détermination des paramètres de «biosphère » [11][15]. Pour les paramètres de transfert des radionucléides simulés par le modèle Aquabios (H-3, I-129, Pu-239) des documents AIEA [16] ont servi de référence. Les paramètres spécifiques au $\mathrm{C}-14$ sont issus de documents relatifs à une inter-comparaison C-14 réalisées par le groupement BIOPROTA [12][13]. 


\section{Résultats de l'analyse multiparamétrique}

\subsection{L'analyse multiparamétrique du transfert par l'eau}

A partir des fonctions de répartition (distribution de probabilité cumulée) des concentrations dans la rivière des Noues d'Amance, pour les quatre radionucléides traités (H-3, C-14, Pu-239, I-129), la variabilité de leur concentration dans le cours d'eau est déterminée.

La variabilité la plus forte est associée au radionucléide présentant la plus forte incertitude sur son activité mesurée dans les effluents du CSA ; le (C-14).

\subsection{Analyse de sensibilité des concentrations dans la rivière des Noues d'Amance}

Le tableau 3 présente les coefficients de corrélation linéaire liant la concentration dans la rivière et les paramètres d'entrée, pour chaque radionucléide traité $(\mathrm{H}-3, \mathrm{C}-14, \mathrm{Pu}-239$ et I-129).

\begin{tabular}{|c|c|c|c|c|}
\cline { 2 - 5 } \multicolumn{1}{c|}{} & \multicolumn{4}{c|}{ Radionucleides } \\
\hline Parametres d'entrée & H3 & $C \cdot 14$ & Pu-239 & $1 \cdot 129$ \\
\hline $\begin{array}{c}\text { Volume du bassin } \\
\text { d'orage }\end{array}$ & 0 & 0 & 0 & 0 \\
\hline $\begin{array}{c}\text { Débit des Noues } \\
\text { d'Amance }\end{array}$ & $-0,22$ & $\cdot 0,15$ & $\cdot 0,34$ & $\cdot 0,26$ \\
\hline $\begin{array}{c}\text { Activité du } \\
\text { radionucleide }\end{array}$ & 0,97 & 0,98 & 0,93 & 0,95 \\
\hline
\end{tabular}

Tableau 2- Coefficients de corrélation linéaire de Pearson entre la concentration dans la rivière et les paramètres d'entrée
L'analyse des résultats indique que c'est l'activité du radionucléide qui est le paramètre d'entrée le plus influent sur la concentration dans la rivière compte-tenu des très faibles valeurs mesurées, ce quel que soit le radionucléide (corrélation positive), et dans une moindre mesure, le débit des Noues d'Amance (corrélation négative). L'incertitude sur le volume de rejet du bassin d'orage n'a aucune influence.

\subsection{L'analyse multiparamétrique d'évaluation de la dose}

Pour chacun des radionucléides, H-3, I-129, Pu-239 et le C-14, les facteurs de corrélation de Pearson entre la dose et chaque donnée d'entrée (inclus la concentration dans les Noues d'Amance) sont calculés. L'analyse des coefficients de corrélations montre que les paramètres pilotant la dose des radionucléides sont :

- Pour le H-3 et le l-129, la concentration dans les Noues d'Amance, les paramètres gouvernant la consommation et l'origine de l'eau ;

- Pour le C-14, la concentration dans les Noues d'Amance, les paramètres gouvernant la consommation, l'origine de l'eau, la géométrie du champ de culture et la fraction de particules atteignant le sol ;

- Pour le Pu-239, le taux de suspension des particules contaminées, le temps de présence sur les champs et in extenso hors zone, ainsi que le débit respiratoire

\section{Conclusions}

L'étude préliminaire de sensibilité multiparamétrique de la dose aux paramètres hors ouvrages et nappe pour la voie de transfert par l'eau a été menée selon différentes étapes successives :

- Une caractérisation des incertitudes des données d'entrée (volume du bassin d'orage, débit des Noues d'Amance et activité des radionucléides) ;

- Une étude multiparamétrique relative au transfert par l'eau, du bassin d'orage à la rivière des Noues d'Amance, avec pour indicateur intermédiaire la concentration des radionucléides dans la rivière :

- Une étude multiparamétrique relative au modèle biosphère d'évaluation de la dose, avec pour indicateur final la dose pour les populations adultes du groupe agricole.

L'estimation des incertitudes sur les données d'entrée conduit aux remarques suivantes : 
- Le volume rejeté issu du bassin d'orage, le volume du bassin d'orage en lui-même et son incertitude sont bien connus du fait de l'instrumentation et de la maîtrise du volume du bassin d'orage. L'estimation des incertitudes a finalement reposé sur les performances des débitmètres et sur la transmission des données à la supervision ;

- Le débit des Noues d'Amance est un paramètre qui est soumis à des variations à la fois journalières et saisonnières. L'estimation des incertitudes sur la valeur moyenne du débit des Noues d'Amance (enveloppant l'incertitude instrumentale et les variations du cours d'eau) est représentée par la moyenne des écarts-types journaliers ;

- Aux incertitudes analytiques des rejets des effluents industriels ont été ajoutées les incertitudes sur la détermination du volume des cuves. La contribution des incertitudes analytiques est très forte en particulier lorsque les activités mesurées sont inférieures ou égales au seuil de décision (SD). Dans ce cas des rejets des effluents industriels, l'incertitude analytique considérée est de 100\%.

En termes de résultats, et sur la base des éléments ci-dessus, l'analyse multiparamétrique d'évaluation de la dose par la voie «eau» présentée dans ce document conduit à dégager les principaux enseignements suivants :

- Concernant l'évaluation de la concentration aux Noues d'Amance, l'analyse multiparamétrique du transfert par l'eau, du bassin d'orage à la rivière, a montré que la concentration dans la rivière était surtout contrôlée par l'activité du radionucléide (effluents industriels), mesurée dans le bassin d'orage. Cette dépendance est à corréler avec la forte incertitude sur la donnée d'entrée (de $45 \%$ à plus de $99 \%$ d'incertitude) ;

- Concernant l'évaluation de la dose :

- Malgré les multiples incertitudes associées au grand nombre de paramètres du calcul de transfert dans la biosphère, l'incertitude globale sur la dose se limite à un ordre de grandeur;

- la concentration dans la rivière des Noues d'Amance contrôle la dose, à l'exception du Pu-239 (identifié comme représentatif des émetteurs $\alpha$ ), . Il faut noter que ce résultat est fortement influencé par une incertitude élevée sur ce paramètre d'entrée. Dans l'ensemble, la voie de contamination par l'eau joue un rôle comparable à celui des plantes et produits agricoles, mais elle n'est quantifiée que par 2 paramètres (consommation et origine de l'eau);

- En ce qui concerne le Pu-239, la voie de transfert par inhalation domine, au travers des paramètres tels que la mise en suspension ou le transfert par le sol.

\section{Références}

[1] Andra (2013) Bilan annuel de surveillance du Centre et de son environnement (CSA INB $\mathrm{n}^{\circ}$ 149) - année 2012; Rapport Andra n SUR.BI.AEES.13.0043

[2] Journal Officiel de la République Française du 21 août 2013, Article 5.3.2

[3] GUM (1995) Guide pour l'expression de l'incertitude de mesure (GUM 1995 avec des corrections mineures); Rapport n JCGM 100:2008.

[4] ANDRA (1999) Gestion des effluents liquides radioactifs de type $B$ et effluents produits sur le CSA et TFB; Rapport n QUA.PR.ADCS.99.5017/F.

[5] Endress Hauser (2008) PV d'étalonnage du débit avec ajustement; Rapport n ref 205066461863178 du 14/02/2008.

[6] ANDRA (2012) Cahier des charges de réaménagement de la station hygrométrique des Noues d'Amances au pont "CD24", 03/04/2012 Rapport n ENV.RP.FEES.11-0037/C.

[7] NF ISO 11929 Détermination des limites caractéristiques (seuil de décision, limite de détection et extrémités de l'intervalle de confiance) pour mesurages de rayonnements ionisants, Principes fondamentaux et applications; Rapport $\mathrm{n}^{\circ} \mathrm{n} / \mathrm{a}$.

[8] Albrecht, A. (2010) Les équations du modèle de transfert du carbone-14 $\left({ }^{14} \mathrm{C}\right)$ dans la biosphère (AquaC_14) et leur intégration dans le code " Modèle Management, MoM "; Rapport Andra n C.NT.ASTR.10.0052.A, version B en préparation

[9] Andra (2004) Centre de l'Aube Rapport de Sûreté Volume III Chapitre 4 Analyse de sûreté relative aux transferts par l'eau; Rapport Andra n SUR RP AEES 04-0019/A.

[10] Albrecht, A. (2007) Les équations du modèle de transfert des radionucléides dans la biosphère (Aquabios) et leur intégration dans le code " Modèle Management, MoM "; Rapport Andra n C.NT.ASTR.06.0036.B, version C en préparation. 
[11] Limer, L.M.C., A. Albrecht, V. Hormann, M.-O. Gallerand, C. Medri, D. Perez-Sanchez, K. Smith, G. Smith, and M. Thorne (2012) Improving Confidence in Long-term Dose Assessments for U-238 Series Radionuclides; Rapport BIOPROTA n n/a.

[12] Smith, K., A. Albrecht, M. Thorne, F. Coppin, A. Ikonen, D. Perez-Sanchez, G. Smith, and L. Limer (2013) Se-79 in the Soil-Plant System Phase 2: Approaches to Modelling; Rapport BIOPROTA $n^{\circ}$ in review.

[13] Limer, L., K. Smith, A. Albrecht, L. Marang, S. Norris, G.M. Smith, M.C. Thorne, and S. Xu (2011) C-14 Long-term dose assessement: Data review, scenario development, and model comparison; Rapport BIOPROTA n ${ }^{\circ}$ CRPFSTR 120002.

[14] Albrecht, A. (2002) CENTRE AUBE Analyse de sensibilité des paramètres affectant le comportement des radionucléides pour le rapport de sûreté 2004; Rapport Andra $\mathrm{n}^{\circ}$ SUR.NT.ASTR.02.095.

[15] Albrecht, A. and M.O. Gallerand (2004) Évaluation des facteurs de conversion biosphère pour les radionucléides et des risques associés aux toxiques chimiques. Modélisation de leur comportement dans les biosphères de référence - Site de Meuse / Haute-Marne HAVL Argile; Rapport Andra n ${ }^{\circ}$ C.NT.ASTR.03.0110.B.

[16] IAEA (2010) Handbook of parameter values for the prediction of radionuclide transfer in terrestrial and freshwater environments; Rapport International Atomic Energy Agency $\mathrm{n}^{\circ}$ 472. 